\title{
TRAVAUX DE LA STATION DE RECHERCHES APICOLES DE BURES-SUR-YVETTE EN 1959
}

\author{
PAR
}

\section{Rémy CHA UVIN}

\section{Pollen et gelée royale.}

Chauvin a publié le compte rendu de ses dernières recherches sur ces substances en lui donnant la forme d'une revue générale. Outre ses propres travaux parus auparavant, il y décrit l'action du pollen sur les cellules sanguines, qui augmentent fortement en nombre chez la souris ayant ingéré du pollen, et aussi bien dans la série rouge que dans la blanche, ce qui doit tenir à une concentration du sang. D'autre part, les hormones hypophysaires (ACTH et STH) bloquent l'action hyperglycémiante du pollen. L'insuline l'annule également; le pollen paraît incapable de faire reprendre la croissance des souris qui l'ont achevée; ce qui le différencie nettement du STH.

Quant à la gelée royale, son action hyperglycémiante, non plus par injection mais par voie buccale, a été mise en évidence (sondage cosophagien chez la souris) ; elle se produit même avec de la gelée royale fraîche n'ayant pas subi de vieillissement préalable (nécessaire au déclenchement de l'hyperglycémie, quand la gelée est administrée par voie sous-cutanée). I es hormones hypophysaires paraissent contrarier dans une mesure mal déterminée l'action de la gelée royale sur le sucre du sang. A l'aide de la réaction d'hyperglycémie, ChaUvis a prouvé la grande fragilité de la gelée pure ou en solution, qui, même à la glacière, ne conserve guère plus d'un mois ses propriétés. La gelée parait dépourvue d'action sur la croissance, sauf à très faible dose, où apparaît une certaine accélération. Chauvin critique aussi une revue tendancieuse publiée par les Johansson où l'exposé des faits concernant la gelée royale est déformé par un parti-pris da négation. Après une dizaine d'années de recherches, on peut dire maintenant que la substance donne de grands espoirs et que nous disposons de tests biologiques suffisamment précis pour permettre ın étalonnage. 


\section{La construction chez les abeilles.}

La thèse de DARCHEN sur la construction chez les abeilles est parue en I959. C'est un très important travail, qu'il est difficile de résumer ici. DARCHEN y insiste beaucoup sur le rôle des chaînes cirières à travers lesquelles circule certainement une "information " qui utilise sans doute les minimes différences de tension musculaire entre les pattes des ouvrières; comme ces pattes sont accrochées les unes aux autres, on conçoit que la grappe puisse "se tenir informée " des perturbations de l'ouvrage ou de la manière dont il progresse. Un autre caractère frappant des constructions, sur lequel DARCHEN insiste très justement, consiste dans les retouches continuelles que les abeilles apportent à leur travail ; non seulement elles peuvent construire, mais elles sont capables aussi de détruire l'ouvrage qui ne correspond pas à certaines normes, et elles ne s'en privent pas. On peut d'ailleurs distinguer dans le rayon en construction, une série de zones qui ne sont pas équivalentes : des zones critiques où l'interposition d'un obstacle est vivement ressentie, se rencontrent à l'extrémité libre du rayon et surtout sur ses bords. Le rôle de la reine enfin est considérable et sa présence facilite énormément la construction, sans doute par une distribution de substances du type de la phérormone.

Les régulations de la construction peuvent atteindre un degré surprenant. Par exemple les abeilles peuvent tordre une languette de cire introduite perpendiculairement à deux rayons, et la rendre parallèle. Elles peuvent aussi si elle est rectangulaire ou carrée, en abattre les angles avant de l'étirer, pour lui donner la forme ovalaire classique du rayon en voie d'extension (DARCHEN, I959). Ces régulations sont tout à fait extraordinaires et DARCHEN se propose d'en faire une étude approfondie. Il a étudié aussi le rôle $d u$ fond des cellules sur la cellule elle-même, à l'aide d'une technique très simple : en détachant les cellules de leur fond à l'aide d'un couteau chauffé introduit parallèlement au fond il peut transporter les tubes cellulaires de mâles sur un " fond d'ouvrières " et viceversa. Dans ce cas, les abeilles transforment la cellule de manière à la raccorder au fond; c'est-à-dire que des cellules de mâles, transposées sur fond d'ouvrières, seront peu à peu à l'aide de mille retouches irrégulières, transformées en cellules d'ouvrières. Ce sont les caractères du fond qui règlent le type de la cellule.

On a signalé de nombreuses fois les difficultés qu'éprouvaient les mellifères solitaires à réparer leur cellule quand elle se trouve percée ; l'abeille maçonne en particulier ne peut le faire qu'à un certain moment. Par contre, chez Apis mellifica, DARchen d'abord sur les cellules d'ouvrières et VUILIAUME ensuite sur les cellules royales, ont montré que les réparations s'effectuaient sans difficulté et très rapidement, que l'expérimentateur les ait pratiquées sur le fond ou sur les parois. 


\section{Le comportement au cours de l'élevage des reines.}

VUILLAUME a continué ses recherches sur l'élevage des reines. Il y a intérêt à greffer les jeunes larves sur une assez forte quantité de gelée royale, car le pourcentage des élevages réussis s'en trouve augmenté (d'ailleurs les ouvrières dégorgent une quantité plus grande de gelée royale quand on ne l'a pas ménagée lors du greffage). I a substitution de larves très jeunes à des larves plus âgées qui se trouvaient déjà dans les cellules royales amène un nouveau et très important dégorgement de gelée sur celle qui s'y trouvait déjà. Si l'on introduit plusieurs larves à la fois dans de larges cupules, l'élevage échoue complètement, que les larves soient côte à côte ou qu'elles soient séparées par des cloisons. La raison de l'échec vient probablement de la forme anormale du fond (trop large) et des bords, à quoi les nourrices sont très sensibles. On peut toutefois greffer deux larves à la fois dans une cupule normale, ce qui incite les abeilles à dégorger davantage de gelée royale; mais une seule larve subsiste à la fin. A condition d'employer comme substrat de la gelée pure ou très concentrée, on peut faire accepter aux nourrices même des larves âgées. Phénomène très intéressant, de nouvelles cupules peuvent être introduites à côté de cupules en voie d'operculation ou même à côté de cellules mûres, en voie de destruction parce que la jeune reine vient d'éclore. L'élevage se poursuit néanmoins, preuve que les stimuli qui poussent les abeilles à détruire les cellules et ceux qui les déterminent à les élever peuvent jouer côte à côte dans la ruche. Vuiliaume avait étudié aussi dans un travail précédent, les substances d'acceptation et d'inhibition de l'élevage; la substance inhibitrice qu'il avait signalée dans certaines cires se confond avec la propolis ou plus exactementavec une essence qu'on peut en retirer dans certaines conditions; quant à la substance d'acceptation elle est entraînable par 1'acétone mais non par le benzène. Il faut apporter certains tempéraments à la théorie de BUTLER suivant lequel la "substance royale " (phérormone) inhibe la construction des cellules royales. On ne peut l'admettre que si l'on entend leur construction "spontanée ". Car si l'on introduit des cupules où se trouvent déjà de jeunes larves, les choses se passent d'une manière bien différente : elles sont acceptées lorsque la reine est emprisonnée à côté d'elles dans une petite cagette fermée par une toile métallique simple (pemettant l'échange de l'hormone avec les ouvrières) et même si la reine est libre (mais une première série est seulement acceptée dans ce cas; on n'obtient plus d'élevage après introduction d'une seconde série de cupules). Cette acceptation de l'élevage royal alors que la reine est présente et libre donne beaucoup à réfléchir ; l'expérience n'a pas été poursuivie jusqu'à l'éclosion des jeunes reines: on peut se demander si l'essaimage ne se déclencherait pas alors. 


\section{Existe-t-il chez Apis des sexués de remplacement?}

Une note très importante de DARCHEN et VUILLAUME remarquel'existence de deux types de cellules royales, les unes qui reposent sur un socle de cire très épais, les autres qui prolongent seulement une cellule d'ouvrière un peu plus large que les autres. Les premières sont construites au cours du renouvellement spontané de la reine par les abeilles (lorsqu'elle est trop vieille par exemple) ou au moment de l'essaimage. I es secondes se développent lorsque la reine disparaît accidentellement d'une manière brutale; dans ce dernier cas les abeillss élèvent des larves spéciales (que DARCHEN et VUILLAUME appellent les " pré-reines") et qui se trouvent toujours au milieu des cadres dans des cellules plus larges ; elles reçoivent aussi davantage de gelée royale et peuvent être transformées en reines à un âge très avancé. Mais elles peuvent très bien ne pas être utilisées : elles fournissent alors des formes intermédiaires qui ont été signalées de nombreuses fois dans les ruches et qui rendent parfois difficiles la recherche de la reine. Peut-être donnent-elles les premières ouvrières pondeuses quand la reine n'a pu être remplacée.

\section{Autres recherches sur le comportement des abeilles.}

VuILIAUME a étudié la rétention mnémonique du gîte chez les ouvrières dont la ruche a été changée de place. Les butineuses exécutent pendant très longtemps un crochet vers l'ancien emplacement au cours de leur vol de retour, et ce comportement dure probablement jusqu'à leur mort. Si bien que l'habituation à l'emplacement serait sans doute indélébile mais pas assez impérieuse néanmoins pour empêcher le retour dans la colonie déplacée. L'" anamnèse " se produit au cours de 1'essaimage. Mais elle n'est qu'apparente, car si l'on enlève la reine, les abeilles reviennent aussitôt à l'ancienne ruche. On induit aussi l'anamnèse en greffant des cupules royales munies d'une jeune reine dans un groupe d'abeilles orphelines placées à quelques mètres de leur ancienne ruche.

DARCHEN a décrit le comportement des abeilles qui nidifient à l'air libre à l'intérieur d'une serre; on peut ainsi réaliser sans aucune difficulté un grand nombre d'observations qui seraient impossibles par toute autre technique. D'autre part, à l'intérieur de la serre, l'essaim peut vivre à l'air libre pendant un laps de temps très prolongé.

\section{Les hormones et les antibiotiques de la ruche.}

LAVIE et Mlle PAIN ont mis en évidence une certaine convergence de leurs travaux respectifs. En effet, l'antibiotique découvert par I/AvIE 
sur le tégument de l'ouvrière adulte, puis sur celui de la reine, présente les plus grandes analogies avec l'ectohormone (phérormone) étudiée par Mlle Parn. Mieux les conditions qui permettent aux ouvrières d'atteindre leur plein développenment ovarien sont réalisées, plus on trouve d'antibiotique dans leur tégument. En présence d'une jeune reine, leurs ovaires diminuent et leur teneur en antibiotique baisse pendant qu'augmente la teneur en antibiotique de la reine ainsi que sa production de phérormone et son pouvoir attractif vis-à-vis des ouvrières. I1 n'existe chez la reine qu'un seul organe qui possède à la fois des propriétés antibiotiques et soit attractif pour les ouvrières : les glandes mandibulaires. Ajoutons que les deux propriétés (antibiotique et attractive) sont en étroite corrélation et que leurs variations sont parallèles; si bien qu'il serait sans doute possible de remplacer les mesures pénibles du pouvoir attractif des reines par une évaluation facile et rapide de son facteur antibiotique. Le développement ovarien des reines, par contre, n'a aucun rapport ni avec leur attractivité, ni avec leur facteur antibiotique.

D'ailleurs, d'après LAVIE, l'intérieur de la ruche est en quelque sorte tapissé de substances antibiotiques qui montrent une certaine activité vis-à-vis des maladies bactériennes du couvain (B. alvei, B. larvae). I1 n'est pas impossible que la présence ou les variations de ces défenses antibactériennes globales ne jouent un rôle dans le développement ou la propagation des maladies des abeilles.

\section{Pollinisation.}

Un département pour l'étude de la pollinisation s'est ouvert à la station de BUREs et a été placé sous la direction de $\mathrm{I}_{\mathrm{E}} \mathrm{ECOMTE}$, assisté de CrocheT. Les premières recherches portent sur un problème devenu depuis peu fort grave, celui de la pollinisation de la luzerne. Elles montrent que dans les conditions habituelles de la métropole, les populations d'abeilles domestiques et celles des autres hyménoptères sont très insuffisantes pour assurer une pollinisation satisfaisante. Il est intéressant de noter que d'après les apiculteurs, les luzernes ne seraient habituellement visitées que dans des régions bien délimitées, où justement on pourrait récolter du miel de luzerne. Cette observation pourrait fournir une direction de recherches fructueuses : il existerait d'ailleurs une corrélation entre le développement moyen des pieds de Luzerne et le nombre d'abeilles au mètre carré : plus la luzerne est haute, plus on y trouve de butineuses. Le phénomène de dérive des abeilles, dans les ruches placées en lignes, paraît très important et il faudra trouver le moyen d'y remédier. 


\section{Techniques apicoles et divers.}

FRESNAYe a étudié d'une manière très détaillée les méthodes de protection $d u$ bois des ruches. La plus pratique semble consister dans un paraffinage à haute température, suivi d'un égouttage soigneux ; mais le paraffinage à basse température tel que l'emploient certains apiculteurs n'est nullement satisfaisant. VuilLAUME décrit aussi une technique qui permet sans manipulations compliquées et en utilisant un matériel standard, de récolter dans la même ruche du miel, de la gelée royale et du pollen. CourTors et $\mathrm{I}_{\mathrm{A}} \mathrm{ECOMTE}$ marquent des butineuses au moyen d'or radioactif mélangé au sirop avec de bons résultats. Ils prouvent aussi que l'ouvrière irradiée au Cobalt 60 supporte sans dommages apparents une dose de 18 ooo r ; elle meurt à 200 ooo r. Leconte et Martouret démontrent la non toxicité pour les abeilles d'une souche de Bacillus thuringiensis utilisée pour la lutte biologique contre les chenilles.

\section{Revues générales.}

DARCHEN présente une revue générale sur l'abeille en tant qu'insecte social; I OUVEAUx sur la technologie du miel; LECONTE sur la pollinisation.

\section{Visites, réunions, participation à des congrès.}

Nous avons reçu la visite de MM. Ben Neriah (Israël), KhalLapur (Inde) et Dyce (U. S. A.).

A la fin de l'année I959 et à l'occasion d'une réunion privée de la Bee Research Association, tenue à Paris, les directeurs des principaux laboratoires européens ont visité la Station de BUREs et ses récents aménagements.

\section{STATION EXPÉRIMENTALE D'APICULTURE DE MON'TFAVE'T}

Les installations de la station sont maintenant achevées et le rucher complètement organisé. Une description très détaillée du système de pasteurisation du miel et de la méthode suivie dans la conduite du rucher sera donnée ici même dans peu de temps. 


\section{LISTE DES PUBLICATIONS DES STATIONS DE BURES ET MONTFAVET EN I959}

(I) Chauvin (R.). - L'action de la gelée royale sur les animaux supérieurs et sur 1'homme; critique de la revue des Johansson. Ann. Abeille, 2, 223-34.

(2) Chauvin (R.). - La valeur diététique et thérapeutique des produits de la ruche. Produits pharmacentiques, I4, no $5,6,7$.

(3) Courtois (G.) et Lecomte (J.). - Marquage d'abeilles au moyen d'or radioactif. Jour. Appl. Rad. Isot., 5, 265-8.

(4) Courtors (G.) et LeComte (J.). - Sur la résistance au rayonnement gamma de l'abeille ouvrière. Ann. A beille, 2, 285-9I.

(5) Darchen (R.). - Les techniques de construction chez Apis mellifica (Thèse Paris). Ann. Sc. Nat. Zool. I2 ${ }^{\mathrm{e}}$ série, II6-209.

(6) DARchen (R.). - Observation et expérimentation sur un essaim nidifiant artificiellement à l'air libre. Ann. Abeille, 2, 5-I2.

(7) Darchein (R.). - Un des rôles des chaînes d'abeilles : la torsion des rayons pour les rendre parallèles entre eux. Ann. Abeille, 2 ; 193209.

(8) Darchein (R.). - Construction et reconstruction de la cellule des rayons d'Apis mellifica. Ins. Soc. IV (I958), 357-7 I.

(9) Darchen (R.). - L'Abeille, insecte social. Journ. Psych. Norm. Pathol., $75-98$.

(io) Darchen (R.) et Vuiliaume (M.). - Deux types de cellules royales chez Apis mellifica. Existe-t-il chez Apis mellifica des sexués de remplacement? Ann. Abeille, 2, I77-I83.

(II) Fresnaye (J.). - Protection du bois des ruches. Le paraffinage. Ann. A beille, 2, 235-56.

(I2) LAVIE (P.). - Action antibiotique de quelques substances provenant de l'abeille ou de la ruche sur Bacillus lavvae et Bacillus alvei. C. $R$. Acad. Sc., 248, 455-57.

(I3) Lavie (P.) et PAIN (J.). - Les rapports entre las substance antibiotique des reines et des ouvrières d'abeilles, le développement ovarien et 1'ectohormone. C. R. Acad. Sc., 248, I587-89.

(I4) Lavie (P.) et PaIN (J.). - Relation entre la substance attractive, le facteur antibiotique et le développement ovarien chez la reine d'Abeille, Apis mellifica. C. R. Acad. Sc., 248, 3753-55.

(I5) Lecomte (J.). - Luzerne et apiculture. Ann. Abeille, I2, 2II-2I.

(I6) Lrcomte (J.). - Premières observations sur le comportement des insectes pollinisateurs de la luzerne. Ann. A beille, 2, 277-285.

(I7) LECOMTE (J.) et MAR'TOURET (D.). - Non toxicité pour les abeilles des traitements à base de Bacillus Thuringiensis souche Anduze. Ann. Abeille, 2, I7I-75.

(I8) Louveaux (S.). - La Technologie du miel. Ann. Abeille, 2, 343-54.

(Ig) PaIN (S.). - Etude de l'apparition de l'attractivité chez les teines vierges d'Abeille. C. R. Acad. Sc., 248, 32 II-2.

(20) Vuillaume (M.). - Nouvelles données sur la psychophysiologie de l'élevage des reines chez Apis mellifica. Ann. Abeille, 2, II3-I38.

(2I) Vuillaume (M.). - Les productions simultanées de miel, gelée royale et abeilles sont-elles incompatibles? Ann. A beille, 2, 27I-7.

(22) Vulllaume (M.) et Naulizeau (G.). - Réparations par les abeilles de brèches effectuées dans des cupules de cire. Ann. Abeille, 2, 26I7 I. 